\title{
Saponin-based Immunoadjuvant OBI-821
}

National Cancer Institute

\section{Source}

National Cancer Institute. Saponin-based Immunoadjuvant OBI-821. NCI Thesaurus.

Code C91726.

A purified, natural saponin isolated from the soapbark tree Quillaja saponaria Molina with potential immunoadjuvant activity. When co-administered with a vaccine, OBI-821 may increase antibody and cytotoxic T-lymphocyte $(C T L)$ responses ag ainst the vaccine's targeted antigen(s). 